\title{
CONSIDERAÇõES EM TÔRNO DAS HYDROMEDUSAE DA REGIÃO DE FERNANDO DE NORONHA *
}

\author{
M. Vannucci
}

A fauna de hidromedusas e hidropólipos da região da Ilha Fernando de Noronha foi coletada durante uma única viagem que durou sete dias apenas e que realizei no mês de janeiro de 1954 . A quantidade de plancton coletado é pequena, de modo que não pode ser considerada como representativa da fauna de Hydrozoa local. Não encontrei Scyphozoa.

$\mathrm{O}$ que se sabe das águas atlânticas dessa região pode ser resumido da seguinte forma: a água é muito pobre em nannoplancton, sendo o índice logarítmico de 3,5 a 4, isto é, de 5.000 a 10.000 indivíduos por $1.000 \mathrm{~m}^{3}$ (Hentschel, 1933); a água é azuil e a termoclina é próxima à superíície, variando pouco a sua posição nas várias estações do ano. A ilha está situada em plena circulação sul-atlântica, sendo a corrente de leste, ou este, constante durante o ano inteiro.

O plancton foi coletado com rêdes abertas e as amostras de água para determinação da salinidade e a temperatura foram tomadas sòmente em superfície. Devem ser considerados com certa reserva os dados relativos às exigências ecológicas das espécies mencionadas a seguir, devido à escassez de dados detalhados na literatura. Tabelas e dados mais detalhados sôbre essas coletas serão publicados num trabalho em preparo, sôbre as hidromedusas das águas brasileiras **.

- Trabalho apresentado em 1955, no I Simpósio Latino-Americano sôbre Plancton, organizado pela UNESCO, em colaboraçå com o Conselho Nacional de Pesquisas e a Universidade de São Paulo.

* Esse trabalho acha-se atualmente (1959) publicado e intitula-se: “On Brazilian Hydromedusae and their distribution in relation to different water masses”. Bol. Inst. Ocean., vol. 8, p. 24-109. 


\section{ESPÉCIES HOLOPLANCTONICAS}

As espécies holoplanctônicas são representadas por Trachymedusae e Narcomedusae, as quais realizam o seu inteiro ciclo evolutivo em suspensão na água. O fato de não possuírem, em seu ciclo evolutivo individual, nenhuma fase bentônica, torna essas medusas grandemente independentes dos fundos, adquirindo com isso, de um modo geral, hábitos tìpicamente oceânicos. Todavia, algumas Trachymedusae não se limitam às águas oceânicas, como por exemplo, Aglaura hemistoma e mais ainda Liriope tetraphylla, esta última habitante usual das águas costeiras da costa do Estado de São Paulo.

Da ordem Trachymedusae foram coletados espécimes pertencentes a duas famílias, as Geryonidae e as Rhopalonemidae. Distinguem-se as Geryonidae das Rhopalonemidae por possuírem quatro ou seis canais radiais e numerosos canais centrípetos. A presente coleção inclui espécimes dos dois gêneros de que é constituída essa família, a saber: Liriope, com simetria quaternária, e Geryonia, com simetria senária.

Da ordem Narcomedusae constam dessa coleção apenas medusas da família Aeginidae caracterizada pela presença de bôlsas gástricas localizadas interradialmente. Tôdas as Narcomedusae presentes pertencem à espécie Solmundella bitentaculata.

\section{Ord. TRACHYMEDUSAE}

\section{Fam. Geryonidae}

\section{Liriope tetraphylla (Cham. \& Eisenh. 1821)}

Essa espécie foi coletada em quase tôdas as estações, sendo, depois de Aglaura hemistoma a mais freqüente; foram coletados 27 indivíduos. Liriope tetraphylla é uma espécie de ocorrência freqüente e abundante nas camadas superficiais da zona circumtropical e tem como limite de distribuição, ao norte e ao sul, as isotermas de $20^{\circ} \mathrm{C}$ (Thiel 1936, p. 53, f. 10), estando a maioria dentro da área delimitada pelas isotermas de $25^{\circ} \mathrm{C}$. Desde a primeira descrição dessa espécie em 1821, foram descritas mais de 26 "espécies" dêsse gênero. A distinção entre elas era feita principalmente pela forma e posição das gônadas, número e desenvolvimento dos canais centrípetos, comprimento do pseudomanúbrio e tentáculos. Como é comum entre as hidromedusas, o comprimento do pedúnculo e dos tentáculos é variável intra-especificamente e depende em grande parte do estado de contração do 
órgão em questão, no momento da fixação. Além disso, a forma e posição das gônadas, assim como o número dos canais centrípetos são caracteres que variam não só intra-especificamente como também com o estado de desenvolvimento individual e nos vários quadrantes de um mesmo indivíduo. À medida que coleções maiores foram sendo realizadas em áreas mais extensas, os autores verificaram que as "espécies" descritas eram, na realidade, ligadas umas às outras por numerosas formas de transição, morfològicamente intermediárias entre as anteriormente consideradas como espécies separadas.

Browne (1926) foi o primeiro autor a verificar que os indivíduos de um mesmo cardume eram, em geral, morfològicamente concordantes enquanto que os de cardumes distintos geralmente diferiam. Todavia (Browne, 1.c.), com um número suficientemente grande de coletas, pôde-se estabelecer uma série de indivíduos que exibem todos os aspectos morfológicos intermediários. Ocorre, portanto, nessa espécie, um gradiente morfológico que apresenta uma distribuição geográfica irregular. Por existirem todos os intermediários entre as "espécies" descritas, creio, portanto, correta a opinião dos autores que recomendam atribuir tôdas as formas descritas a uma única espécie, a qual, por motivos de prioridade, deve chamar-se Liriope tetraphylla.

Utilizando os dados que pude reunir da literatura, sobretudo aquêles compilados por Mayer (1910), procurei subdividir essa espécie em raças geográficas, mas não consegui chegar a nenhum quadro satisfatório. Creio, portanto, tratar-se realmente de uma espécie muito variável mas que não está diferenciada em subespécies, provàvelmente devido a suas propriedades euritérmicas e euri-halinas e à falta de barreiras bem delimitadas no pelagial.

Provisòriamente, pode-se explicar a grande semelhança dos indivíduos de um mesmo cardume, pela origem comum de todos êles, de um número reduzido de espécimes ancestrais. Em outros têrmos, o grande número de ovos postos e o desenvolvimento rápido (seis dias até a formação completa da larva ou medusa jovem, seg. Russell 1953, p. 424, autor que, porém, não indica a temperatura ambiente), podem levar à formação de um grande número de descendentes a partir de um pequeno número de indivíduos paternos. Os enormes cardumes de milhares de indivíduos assim originados, evidentemente são constituídos por uma população genèticamente bastante homogênea. Por essa mesma razão, cardumes separados diferirão sensìvelmente uns dos outros. Por outro lado, a cruzabilidade entre indivíduos de populações distintas é demonstrada pela existência das formas intermediárias. A falta de barreiras geográficas e ecológicas impede a formação definitiva de raças ou mesmo de espécies separadas. 
Dos 27 indivíduos dessa espécie, coletados em Fernando de Noronha, 14 são larvas $(52 \%)$; 9 são jovens $(33 \%)$; e sòmente 4 são maduros $(15 \%)$. Não encontrei relação entre a distribuição batimétrica e a idade individual.

Os presentes indivíduos de Liriope tetraphylla são da forma descrita sob o nome de "Liriope exigua", típica das Antilhas, Atlântico equatorial e Mediterrâneo. Apenas um espécime, da amostra $\mathrm{M}_{19}$, uma fêmea com ovos maduros, com $4 \mathrm{~mm}$ de diâmetro, é do tipo descrito sob o nome de "Liriope eurybia".

Os exemplares dêsse material, com $2 \mathrm{~mm}$ de diâmetro, já apresentam um pedúnculo cujo comprimento é igual ao raio da umbrela. O pseudomanúbrio alonga-se regularmente com o crescimento da medusa, assim que essa sai do estado larvário, não ultrapassando, porém, o comprimento de 2,5 vêzes o raio da umbrela.

Espécie estenoterma de águas quentes e euri-halina.

\section{Geryonia proboscidalis (Forsk. 1775)}

Foi coletado apenas um exemplar dessa espécie, em superfície, numa temperatura de $29^{\circ} \mathrm{C}$. $\quad \hat{\mathrm{E}}$ uma espécie rara mas presente em todos os mares quentes; é freqüente sòmente no Mediterrâneo. Thiel (1936, p. 56) indica como limite de sua distribuição a isoterma de $15^{\circ} \mathrm{C}$. ́́ provàvelmente uma espécie estenoterma, de águas quentes, associada às camadas superficiais.

\section{Fam. Rhopalonemidae}

\section{Rhopalonema velatum Gegen. 1856}

Em freqüência, essa é a terceira espécie dessa coleção, tendo sido coletada em oito estações, num total de 25 indivíduos.

Distingue-se da única outra espécie do gênero - $R$. funerarium - por possuir um processo cônico na exumbrela que aparece quando o indivíduo tem cêrca de $1,8 \mathrm{~mm}$ de diâmetro umbrelar. As gônadas começam a se desenvolver em indivíduos de 4,5 a 5,5 mm de diâmetro. Alimenta-se de copépodos e apendiculárias.

Rhopalonema velatum é espécie de águas quentes, com distribuição circumtropical e circumsubtropical, confinada à área delimitada pelas isotermas de $15^{\circ} \mathrm{C}$ ao norte e ao sul (Thiel 1936, p. 15), sendo que a maioria encontra-se na região compreendida 
entre as isotermas de $25^{\circ} \mathrm{C}$. Por via de regra, em águas tropicais Aglaura hemistoma é espécie muito mais freqüente que Rhopalonema velatum; todavia, no Mediterrâneo parece reinar a relação inversa, sendo, segundo Kramp (1924, p. 25), Rhopalonema velatum, nesse mar, a mais comum de tôdas as medusas. A diferença principal entre as duas espécies do gênero reside na distribuição batimétrica, pois que Aglaura habita pequenas profundidades, enquanto Rhopalonema é mais freqüente em profundidades de 20 a $260 \mathrm{~m}$.

Talvez a subida de Rhopalonema para águas mais superficiais no Mediterrâneo, seja devida à alta salinidade das camadas superiores, nesse mar. Thiel (1936, p. 13) encontrou, no material coletado pelo "Meteor", uma maioria de indivíduos pequenos e imaturos. A escassez de adultos tanto no material do "Meteor" como no presente, pode, talvez, ser explicada pela morte precoce dos animais depois de alcançarem a maturidade sexual.

É uma espécie estenoterma de águas quentes e provàvelmente esteno-halina.

\section{Aglaura hemistoma Pér. \& Les. 1809}

Essa espécie é a que foi coletada em maior número, alcançando um total de 145 indivíduos. Estêve presente em tôdas as estações afora $\mathrm{M}_{3}, \mathrm{M}_{6}, \mathrm{M}_{11}, \mathrm{M}_{12}, \mathrm{M}_{17}$, em superfície; $\mathrm{M}_{7}$ a $10 \mathrm{~m}$ de profundidade e $\mathrm{M}_{18}$ a $20 \mathrm{~m}$ de profundidade. dantes.

Essa espécie é uma das Trachymedusae pelágicas mais abun-

As principais conclusões provisórias a que pude chegar, estudando as medições e contagens que realizei com o material coletado em Fernando Noronha, são as seguintes, quanto à distribuição: 1 - grande maioria dos indivíduos ocorre entre 10 e $30 \mathrm{~m}$ de profundidade; 2 - faltam na maioria das estações superficiais; além disso são escassos entre 30 e $50 \mathrm{~m}$ de profundidade (não foram realizadas coletas em profundidades maiores); $3-$ dos 145 indivíduos coletados, apenas 8,5\% dos mesmos apresentam gônadas grandes; 4 - todos os indivíduos maduros estavam compreendidos entre a superfície e $10 \mathrm{~m}$ de profundidade.

Essa espécie reproduz-se o ano inteiro; segundo Thiel (1936, p. 42), habita as águas compreendidas entre as isotermas de $20^{\circ} \mathrm{C}$. Já foi encontrada em profundidades superiores a $600 \mathrm{~m}$.

É difícil explicar a escassez de adultos, pois eu mesma encontrei anteriormente, em superfície, no Banco Jaseur (Vannucci 1954, p. 119), uma grande maioria de indivíduos adultos; além disso, é 
pouco provável a descida dos animais maduros para profundidades maiores, pois todos os indivíduos sexuados dessa coleção foram capturados entre a superfície e $10 \mathrm{~m}$. É, portanto, possível que as medusas dessa espécie morram logo após libertarem os gonócitos. Pelo aspecto do histograma, com grande porcentagem de indivíduos de tamanho médio, parece-me poder concluir que se trata de uma população homogênea, havendo uma parada de crescimento na época em que começam a se desenvolver as gônadas.

Em águas frias, $A$. hemistoma é substituída por uma espécie muito semelhante: Aglantha digitale que difere de Aglaura por ter gônadas nos canais radiais em vez de as ter no pseudomanúbrio.

\section{Ord. NARCOMEDUSAE}

\section{Fam. Aeginidae}

Solmundella bitentaculata Quoy \& Gaymard 1833

Dessa espécie encontrei sòmente dois exemplares nas estações $\mathrm{M}_{10}$ e $\mathrm{M}_{13}$, respectivamente a 40 e $10 \mathrm{~m}$ de profundidade.

É uma espécie vastamente distribuída, provàvelmente cosmopolita, euritérmica, euríbata e provàvelmente euri-halina: já foi encontrada até a $2.600 \mathrm{~m}$ de profundidade.

Dêsse gênero, foram descritas duas “espécies”, atualmente consideradas sinônimos, o que, porém, ainda não está definitivamente estabelecido. As duas formas são macrogeogràficamente simpátricas.

\section{ESPÉCIES MEROPLANCTÔNICAS}

As espécies meroplanctônicas são as representadas por Anthoe Leptomedusae providas de uma fase bentônica fixa durante o seu ciclo evolutivo. Êsse fato torna as espécies meroplanctônicas dependentes de fundos próximos; de fato, são geralmente formas costeiras ou de águas rasas. É por essa razão que, sendo possível e, por vêzes, até comum encontrar-se espécies holoplanctônicas em águas costeiras, é extremamente raro e devido a correntes especiais ou excepcionais, encontrar-se espécies meroplanctônicas em águas oceânicas.

Da ordem Anthomedusae foi coletado sòmente um espécime pertencente à família Corynidae, Sarsia sp. e alguns pólipos do gênero Syncoryne, que é o gênero ligado por relações metagenéticas com as medusas do gênero Sarsia. 
Da ordem Leptomedusae foram capturados sete espécimes de Eutima mira, família Eutimidae; quatro espécimes de Obelia. possivelmente Obelia geniculata, um indivíduo de Phialidium sp. e um fragmento de Clytia cylindrica, que é o hidróide ligado a Phialidium (medusa), por metagênese, da família Campanularidae. Também foram coletados pelas rêdes de plancton, vários fragmentos de colônias de hidróides tecados, pertencentes a gêneros que não produzem medusas e cuja fase vagil é apenas representada por uma plânula de curta duração.

As espécies meroplanctônicas são, portanto, representadas tanto pelas fases livremente natantes (medusas) como pelas fases bentônicas (pólipos), em alguns casos havendo representantes das duas fases do mesmo gênero, provàvelmente até da mesma espécie (Sarsia e Syncorine; Phialidium e Clytia). É de se notar ser excepcional a presença de formas bentônicas em amostras de plancton.

\section{Fam. Eutimidae}

Eutima mira McCrady 1857

Encontrei, ao todo, sete indivíduos dessa espécie, coletados nas estações $M_{12}$ e $M_{13}$, em superfície e a $10 \mathrm{~m}$ de profundidade. É uma espécie de superfície. Não encontrei os pólipos dessa espécie.

\section{ESPÉCIES BENTONNICAS}

Encontrei nas amostras de plancton fragmentos pertencentes a quatro espécies diferentes de Hydrozoa da família Plumulariidae, todos desprovidos de fase medusóide, a saber: Monotheca margaretta f. typica, Plumularia inermis, Aglaophenia contorta, A. pluma. Na zona intercotidal, recolhi colônias pertencentes às seguintes espécies: Schizotricha billardi, também da família Plumulariidae; Dynamena crisioides f. typica, da família Sertulariidae.

\section{CONCLUSÕES}

Pela mera leitura da lista de espécies coletadas nas águas de Fernando de Noronha, saltam aos olhos duas características bem marcadas: a grande preponderância de espécies oceânicas e a relativa abundância de espécimes ou fragmentos de formas bentônicas coletados com rêdes de plancton. 
Ambos os fatos são fàcilmente explicáveis:

1 - A posição geográfica da ilha, distante 175 milhas do limite leste da plataforma continental da América do Sul e a cêrca de 195 milhas do litoral mais próximo, assim como o fato de estar situada no seio da grande circulação sul-atlântica, tornam evidente dever a fauna planctônica ser essencialmente oceânica nessa região. Assim, das nove espécies diferentes coletadas, cinco são tìpicamente oceânicas; quatro das Trachymedusae e uma das Narcomedusae, tôdas holoplanctônicas, desprovidas de fase bentônica no seu ciclo. As quatro espécies restantes são representadas por três Leptomedusae e uma Anthomedusae, tôdas meroplanctônicas, com uma fase bentônica no seu ciclo reprodutivo individual. Quanto ao número de indivíduos, 200 , isto é, $93,9 \%$ pertencem a espécies holoplanctônicas e apenas 13, isto é, 6,1\% a espécies meroplanctônicas. $\quad \hat{\mathbf{E}}$ bem verdade que nem tôdas as espécies holoplanctônicas são também exclusivamente oceânicas; de fato Liriope tetraphylla é muito comum em águas costeiras, até mesmo em regiões de salinidade baixa (Vannucci 1951, p. 91). Verifica-se assim que mesmo dentre as espécies holoplanctônicas, Liriope tetraphylla não é exclusivamente oceânica; o mesmo é possível dizer quanto a Aglaura hemistoma que, se bem que seja uma espécie oceânica, pode, ocasionalmente, ser encontrada também na região nerítica, em pequeno número de indivíduos. Rhopalonema, Solmundella e Geryonia, pelo contrário, são tìpicamente oceânicas. O número de indivíduos de Liriope é de apenas $12,5 \%$ do número total de espécies holoplanctônicas, enquanto que Aglaura hemistoma é a espécie mais abundante: 145 indivíduos, isto é, $72,5 \%$ do total de indivíduos holoplanctônicos capturados.

2 - O número relativamente grande de fragmentos de hidróides em suspensão na água, em grande parte ainda vivos, sòmente pode ser explicado da seguinte maneira: o forte impacto das águas da corrente, batendo sôbre a plataforma insular quebra e arranca fragmentos de colônias que são, então, levados para camadas mais superficiais pela própria corrente ascendente que resulta dêsse impacto. Trabalhando manualmente na zona intercotidal da ilha, consegui colecionar amostras de apenas duas espécies bentônicas: Dynamena crisiodes f. typica e Schizotricha billardi, ambas desprovidas de fase medusóide natante.

Em tôdas as estações, a água de superfície teve salinidade compreendida entre os extremos seguintes: $36,07 \%$ e $36,14 \%$; e temperatura compreendida entre os extremos seguintes: $27,1^{\circ} \mathrm{C}$ e $29^{\circ} \mathrm{C}$. 


\section{S U M M A R Y}

During a seven days trip to the oceanic island of Fernando de Noronha, plankton was hauled from depths varying between $50 \mathrm{~m}$ and the surface. All the nets used were open nets, one was $1 \mathrm{~m}$ wide at the mouth, the other $25 \mathrm{~cm}$ wide. Due to lack of suitable equipment only the surface temperature and salinity were taken.

Five holoplanktonic species were collected, being Aglaura hemistoma the most abundant (145 specimens) and present at almost every station. Next in frequency of specimens and number of stations are: Liriope tetraphylla (27 specimens), Rhopalonema velatum (25 specimens), Solmundella bitentaculata (2 specimens) and finally Geryonia proboscidalis (1 specimen). Most of the specimens were immature.

Of the meroplanktonic species, the most abundant was Eutima mira ( 7 specimens), next Obelia (perhaps Obelia geniculata, 4 specimens), Phialidium sp. (the medusa of the hydroid Clytia, 1 specimen), a fragment of Clytia cylindrica (hydroid), Sarsia sp. (the medusa of the hydroid Syncoryne, 1 specimen) and a few hydroids of Syncoryne sp. Numerous fragments of thecate hydroids, some taken alive, some not, were also present in the samples.

On the whole, the present collection is characterized firstly by a great majority of oceanic forms both in number of species and number of individuals and secondly by relative abundance of benthonic specimens in the plankton.

The author believes that the first fact may be explained by the geographical position of the island, placed 175 miles away from the edge of the continental shelf or north-eartern Brazil. The second fact might be explained by the strong impact of the South Atlantic current on the insular shelf that may be strong enough to tear fragments from the benthonic hydroid colonies. Due to the impact upon the island's submerged portion, upwelling or strong turbulence might insue, responsible for the presence of benthonic animals in the plankton. Benthonic nematodes were also present.

\section{B I B L I O G R A F I A}

Browne, E. T.

1926. Zoological results of the Cambridge Expedition to the Suez Canal, 1924. V. Report on the Medusae. Trans. Zool. Soc. London, vol. $22, \mathrm{n}^{\circ} 14$, p. 105-115.

HENTSCHEL, E.

1933. Das Pelagial der Wasserschicht. Deutsche Atl. Exp. "Meteor", vol. 11 , part 1 , fasc. 1 , p. 1-168.

Kramp, P. L.

1924. Medusae. Dan. Ocean. Exp. 1908-10 Medit. and adj. Seas, vol. 2, H. 1, 67 p.

Mayer, A. G.

1910. The Medusae of the world. The Hydromeduse. Carnegie Inst: Washington, vol. I and II. $\mathrm{XV}+\mathrm{XV}+498$ p., 55 pls.

THIEL, M. E.

1936. Systematische Studien zu den Trachylinae der Meteor Expedition, zugleich ein Beitrag zu einer Revision der Trachylinae. Zool. Jahrb. Syst., vol. 69, p. 1-92. 
Russell, F. S.

1953. The Medusae of the British Isles. Cambridge University Press. $530 \mathrm{p} ., 35 \mathrm{t}$.

VANNUCCI, M.

1951. Hydrozoa e Schyphozoa existentes no Instituto Paulista de Oceanografia. I. Bol. Inst. Paul. Ocean., vol. 2, n 1, p. 67-98.

1954. Hydrozoa e Schyphozoa existentes no Instituto Oceanográfico. II. Bol. Inst. Ocean., vol. 5, p. 95-149. 